\title{
PSYCHOGENITY OF THE HUMANIZED ENVIRONMENT
}

\author{
Sergiy Boltivets \\ Development State Institute for Family and Youth Policy, Kiyv, Ukraine \\ E-mail: boltivetssergij@i.ua
}

The future of psychological science as we see it depends on our ability to understand the phenomenon of psychogenity of man and of the groups of people in their significance to himself and to every other human being. Psychogenity as we define it, is an ability of the humanized environment to create, reflect, transform, save and expose the constellation of psychic manifestations of the own natural essence of every man as a psychological unity. We consider the humanized environment in the sense broader than that of the social environment as we keep in mind not only the mutual influence of people, but also the fact that human life is mediated by the human environment. The latter is one of the most important components equilibrating human psychogenity, the world exists for man only in its humanized sense.

Psychogenity is the phenomenon of joint mental activity of humans forming mental interplay between them. In this interference is inherent a certain degree of mental strain that arises as a representation of man's energetic potential expression. Mental strain, which is based on the "emotional stress" phenomenon substantiated by Hans Seyle, implies the certain measure of expression of the mental activity of a person as a living being. Just the same degree of mental strain is necessary and exists in any act of interpersonal contact. Without the strain, it just wouldn't be. Mental strain is a measure of psychogenity energy manifestation, important for all sides of the interpersonal interaction. In this sense, psychogenity is an integral phenomenon, an aggregate characteristic independent of the degree of each side energy input.

Such approach justifies itself when analyzing what psychogenity of microsocium or of certain social environment means for each individual, person. At the same time, psychogenity can act as an existential characteristic of the effect produced by the individual himself, by the person, and by the others. In this case, psychogenity may be viewed as a single property of the individual, that is, as a personal psychogenity.

Based on such approach we singled out the psychogenity dominant values for the group to which a person is attached mentally and those of the person in her age changeability. This made it possible to define two correspondent psychogenity dominants - referential and age-related.

The essential difference between these two dominants is their directedness. Referential psychogenity is viewed by us as the total mental strain of the significant to the person microsocium in its meaning for the individual, for the person and her perception. Age-related psychogenity has an opposite direction, oriented towards socium, towards the reference group; it is measured relative to the overall level of referential psychogenity: the latter dominates the former or is inferior to it in terms of mental strain level. Thereby age-related psychogenity parameters are constituents of referential psychogenity of the group, of microsocium under the condition that the bearers belong to above entities.

Methodological basis of development of the technique to estimate age-related psychogenity was the position put forward by us that its directedness is different from that of referential psychogenity. This difference lies in its following essential properties:

a) age psychogenity parameters are constituents of referential psychogenity; 
b) age psychogenity as an aggregate mental strain of a person at a certain stage of her age may be singled out in socium, a referential group only as a difference with the general level of referential psychogenity;

c) age psychogenity taken by itself is a correlate of personal psychological time for which the most significant is the interrelation between the perception of herself as of today and personally perceived images of own psychosocial and psychophysiological experience including its phylogenetic components.

Observing each individual and her identity as a self-sufficient integrity, we at the same time took into account the fact that each such integrity has inherent individually expressed psychogenity as a factor. So each person is seen by us as a separate psychogenetic factor. This made it possible to apply a quantitative indicator to characterize groups of individuals who are in a particular social interaction, and besides who are collected simultaneously. The procedural dimension of the psychosocial interaction is measured in this case with the help of the time factor and doesn't take into account its intensity. formula:

Thus, the method of establishing background psychogenity values involves the following

$$
A x B=C,
$$

where $A$ - is the group members number, $B$ - interaction time, $C$ - background psychogenity factor.

Establishing age-related psychogenity indicator makes it possible to specify personal status in inter-age hierarchy of psychosocial influences and together with the background psychogenity index to make correspondent psychohygienic assessment of the social environment in its significance for the individual.

The age-related psychogenity indicator can be determined with the following developed by us formula:

$$
C=\frac{a_{1}+a_{2}+\ldots+a_{n}}{n},
$$

where $a$ - is the age-related psychogenity indicator of the $i$-th number of the microsocial group ( $i=$ $1,2 \ldots, n) ; \mathrm{n}$ - number of the microsocial group members; $C$ - mean value of the age psychogenity of all members of the microsocial group.

The difference between the mean relation of age-related psychogenity of all microsocial group members and the age psychogenity indicator of the person under study is calculated by the formula:

$$
D=c-a_{i}
$$

where $D$ - is the age psychogenity indicator as it is established for the person.

Thus psychogenity is a fundamental property of the humanized environment expressed in the synthesis of psychosocial interactions of an individual. An essence of psychological assessment lies in tracking individual parameters of social-psychological optimum of person's life activity. This optimum determines the psychohygienic focus of the assessment - to preserve the mental balance of the influences and then - the mental health. 